\title{
High-resolution ultrasonic spectroscopy
}

\author{
V. Buckin, M.Caras Altas \\ School of Chemistry, University College Dublin, Dublin, Belfield, Dublin 4, Ireland \\ vitaly.buckin@ucd.ie
}

\begin{abstract}
:
High-resolution ultrasonic spectroscopy (HR-US) is an analytical technique for direct and nondestructive study of intrinsic properties of liquids and semi-solid materials based on precision measurements of ultrasonic velocity and attenuation. The application areas of HR-US in research, product development, quality and process control include analysis of conformational transitions of polymers, ligand binding, self-assembly and aggregation, crystallization, gelation, analysis of phase transitions and phase diagrams, monitoring of chemical and biochemical reactions. The HR-US measurements can be performed in small sample volumes (down to droplet size), broad temperature range, at ambient and elevated pressures, in various measuring regimes such as automatic temperature ramps, titrations and measurements in flow.
\end{abstract}

Key words: ultrasonic spectroscopy, HR-US, ultrasonic velocity, ultrasonic attenuation, molecular processes.

\section{Introduction}

High-resolution ultrasonic spectroscopy is a relatively novel technique for direct and nondestructive analysis of molecular and microstructural processes in liquids and semisolid materials (e.g. gels) based on precision measurements of ultrasonic properties of materials. Although ultrasonic spectroscopy was utilised for material analysis for a long time and has demonstrated a number of successful applications [1], the capability of this technique in analysis of a broad range of molecular processes has been limited by a number of factors. These included limited resolution/precision in measurements of ultrasonic parameters, large sample volumes required and often complicated measuring procedures. Development of high-resolution ultrasonic spectroscopy (HR-US) based on advances in the modern principles of ultrasonic measurements, electronics and digital signal processing allowed to overcome these limitations [2].

\section{Measuring parameters}

The two major parameters measured in highresolution ultrasonic spectroscopy are ultrasonic attenuation and ultrasonic velocity. Attenuation is determined by the energy losses in the compressions and decompressions in ultrasonic waves, which includes absorption and scattering contributions. This parameter provides information of fast (ultrasonic frequency range) dynamics of molecular processes and microstructural (down to several $\mathrm{nm}$ scale) organisation of materials. As measurements of attenuation do not require high stability of temperature of the medium, they can be performed in large samples and therefore relatively precise (typical precision is $1 \%$ of the measured attenuation). Therefore, attenuation was the parameter responsible for the large portion of the applications of ultrasonic spectroscopy in material analysis in the past. These included kinetics of fast chemical reactions, and particle sizing in emulsions and suspensions, and such processes as aggregation, creaming and sedimentation.
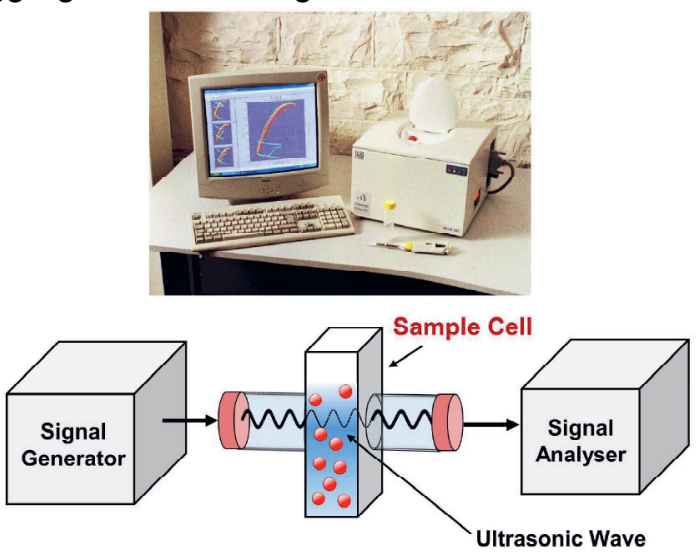

Fig. 1. High-resolution ultrasonic spectroscopy (HR-US) principles of operation.

The major contributor to ultrasonic velocity is the elasticity (compressibility) of the medium. Compressibility is extremely sensitive to the 


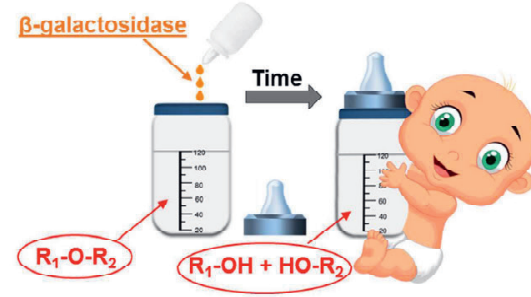

Fig. 2 Real-time ultrasonic profiles of hydrolysis in infant milk at different conditions. Main frame: hydrolysis at 5 ${ }^{\circ} \mathrm{C}, 20^{\circ} \mathrm{C}, 35^{\circ} \mathrm{C}, 40^{\circ} \mathrm{C}$ and $50^{\circ} \mathrm{C}$ at enzyme concentration $3.42 \mathrm{UU} / \mathrm{g}$. Insert: hydrolysis at $20^{\circ} \mathrm{C}$ and enzyme concentrations $5.59,3.42$ and 1.39 $\mathrm{UU} / \mathrm{g}$. One ultrasonic activity unit, UU, represents the amount of enzyme, which hydrolyses $1 \mu \mathrm{mol}$ of $\beta$ galactosidic bonds in Cow\&Gate First Infant milk per min at $20{ }^{\circ} \mathrm{C}$ and reaction extent equal to zero [6].

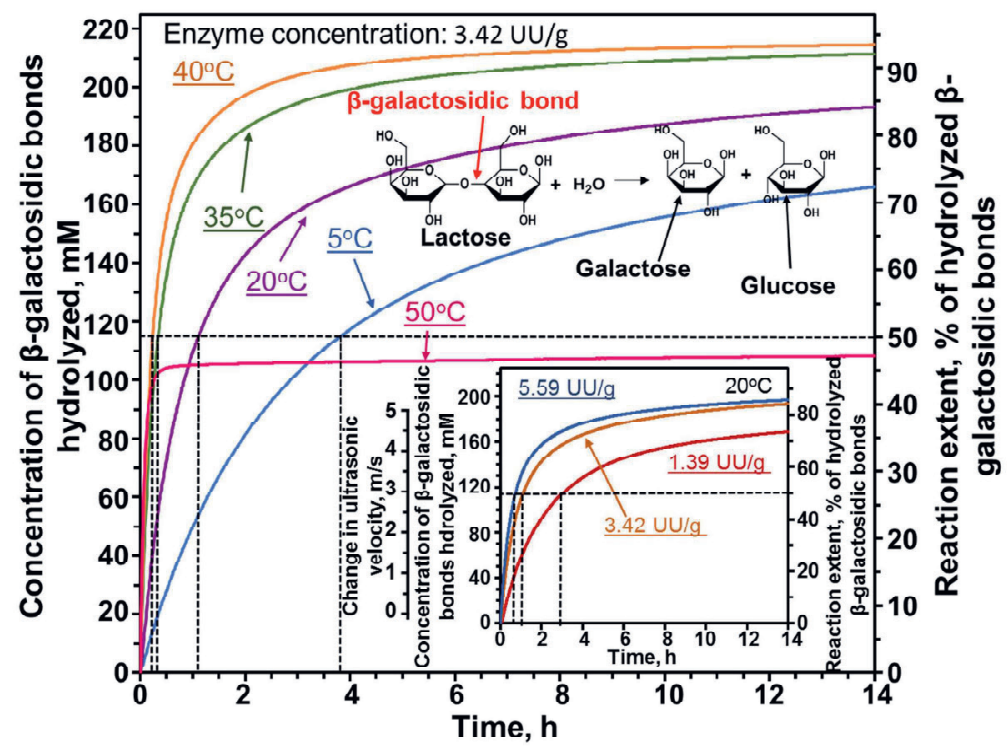

Adapted with permission from Caras Altas, M., Kudryashov, E. \& Buckin, V., Anal. Chem., 2016, 88(9), pp 4714-4723. Copyright (C) 2016 American Chemical Society.

molecular organisation and intermolecular interactions in the medium and can be used to analyse a broad range of molecular processes. However, its application needs high precision of the measurements, which is difficult to achieve in samples significantly exceeding $1 \mathrm{~mL}$ size because of the pure temperature control $\left(10^{-4}\right.$ ${ }^{\circ} \mathrm{C}$ is required) due to temperature gradients in large volumes. The typical precision of ultrasonic spectrometers in measurements of ultrasonic velocity is on the level of 0.1 to $1 \mathrm{~m} / \mathrm{s}$. To overcome this limitation a number of research groups focussed their efforts on ultrasonic velocimeters, e.g. the devices for measurements of ultrasonic velocity in small (1 $\mathrm{mL}$ range) volumes at one frequency or in a very narrow frequency range utilising the singaround [3] or resonator techniques [4,5].

\section{Key attributes of HR-US instruments.}

HR-US instruments employ novel principles of measurements of ultrasonic parameters based on a combination of resonator technique with digital electronic vector analysis. The effective path length travelled by the ultrasonic wave in the sample exceeds the size of the HR-US cell (sample chamber), thus enabling ultrasonic measurements with exceptionally high precision, down to $0.2 \mathrm{~mm} / \mathrm{s}$ for ultrasonic velocity, in a broad range of sample volumes, $0.03 \mathrm{~mL}$ (droplet size) to several $\mathrm{mL}$, with a typical frequency range 1 to $20 \mathrm{MHz}$.

In addition, the geometry of HR-US ultrasonic cells is optimised for easy filling, refilling, cleaning and sterilisation. They can accommodate aggressive liquids such as strong acids or volatile organic solvents, without evaporation during a course of measurements. HR-US measurements can be performed in a wide range of temperatures $\left(-40\right.$ to $\left.130{ }^{\circ} \mathrm{C}\right)$ at ambient or elevated pressures in static and flow-through regimes, and in different media ranging from dilute solutions to semisolid materials. The measurements can include the automated precision titrations of the analysed liquids with a titrant, and also programable temperature ramps for temperature profiling.

\section{Applications of HR-US}

The application area of HR-US in research, product development, quality and process control includes analysis of conformational transitions of polymers, ligand binding, selfassembly and aggregation, crystallization and gelation, analysis of phase transitions and phase diagrams, monitoring of chemical and biochemical reactions and others. Some examples of these applications are outlined below.

\section{Biocatalysis}

Figure 2 illustrates ultrasonic assessment of the impact of temperature and concentration of enzyme on performance of enzyme $\beta$ galactosidase in formulations for reduction of levels of lactose in infant milks [6]. These formulations are added to infant's milk bottles prior to baby feeding to overcome the frequently observed intolerance to lactose (a milk sugar, disaccharide consisting of galactose and glucose linked by $\beta$-galactosidic bond), a serious issue in health development of infants. They provide hydrolysis of lactose to galactose and glucose, which are absorbable in the small intestine. A small amount of enzyme ( $\beta$ - 
galactosidase from Kluyveromyces lactis) solution was added to a $1 \mathrm{~mL}$ ultrasonic cell of HR-US 102 ultrasonic spectrometer loaded with infant milk (Cow\&Gate First Infant milk). The hydrolysis of $\beta$-galactosidic bonds between galactose and glucose after addition of the enzyme, causes a change in ultrasonic velocity (see insert) due to a different hydration level of the products and of the reactant [7]. This change in velocity is recalculated into the concentration of $\beta$-galactosidic bonds hydrolyzed using ultrasonic calibration described earlier [6]. The ultrasonic measurements were collected in the frequency range 2 to $15 \mathrm{MHz}$. As no significant effects of frequency on the ultrasonic reaction profiles were observed, only the data at $5 \mathrm{MHz}$ are presented. No significant changes of ultrasonic attenuation were observed during the hydrolysis, thus, indicating an absence of effects of hydrolysis on microstructural characteristics of milk at all temperatures.

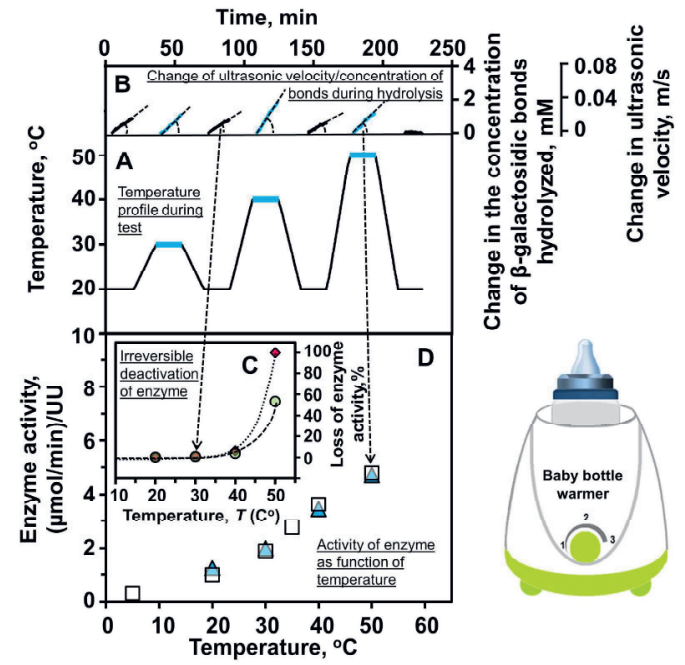

Fig. 3. Effect of temperature on the rate of hydrolysis and irreversible deactivation of enzyme. (A) Temperature in Cow\&Gate First Infant Milk during single sample programmed temperature profiling. (B) Ultrasonic velocity profiles at time intervals of constant temperature. The concentration of enzyme was $0.042 \mathrm{UU} / \mathrm{g}$. (C) Irreversible loss of enzyme activity with temperature. Diamonds: the fractions of deactivated enzyme measured at subsequent (to the indicated temperatures) $20{ }^{\circ} \mathrm{C}$ time intervals, and presented as \% of activity at the precluding $20{ }^{\circ} \mathrm{C}$ interval. Circles: the fractions of deactivated enzyme at each elevated temperature, calculated as the average of the fractions for the precluding and the subsequent $20^{\circ} \mathrm{C}$ interval. (D) Triangles: the effect of temperature on activity of active enzyme (calculated from the slopes ultrasonic velocity vs time, B). Squares: the enzyme activities obtained from separately measured reaction profiles (Fig. 2) [6]. Adapted with permission from Caras Altas, M., Kudryashov, E. \& Buckin, V., Anal. Chem., 2016, 88(9), pp 4714-4723. Copyright (C) 2016 American Chemical Society.
Figure 3 illustrates ultrasonic analysis of effects of temperature on specific activity of the enzyme $\beta$-galactosidase in milk measured in a 'single sample' programmed temperature profiling [6]. The measuring and the reference cells of HR-US 102 spectrometer were filled with $1.1 \mathrm{~mL}$ of infant milk. After equilibration at temperature $20{ }^{\circ} \mathrm{C}, 2 \mu \mathrm{L}$ of $\beta$-galactosidase from Kluyveromyces lactis (aqueous solution, $21.6 \mathrm{UU} / \mathrm{g}$ ) were added to the ultrasonic measuring cell. After the stirring of the sample using a mechanical mini stirrer for $30 \mathrm{~s}$, the temperature profile $(20 \mathrm{~min}$ time intervals at 20-30-20-40-20-50-20 ${ }^{\circ} \mathrm{C}$, Figure $3 \mathrm{~A}$ ) was activated. The measured ultrasonic velocity vs time profile (Figure 3B) provided the concentration of $\beta$-galactosidic bonds hydrolyzed, and the reaction rates (calculated from the slopes ultrasonic velocity vs time). This was used to analyze the effect of temperature on the specific activity of enzyme (D) and to quantify the irreversible effects of temperature on the enzyme (the fractions of deactivated enzyme, C).

The results presented in Figures 2 and 3 were utilized in optimization of the efficiency of commercial enzyme formulations for lactoseintolerant infants.

\section{Self-assembly of protein nanoparticles.}

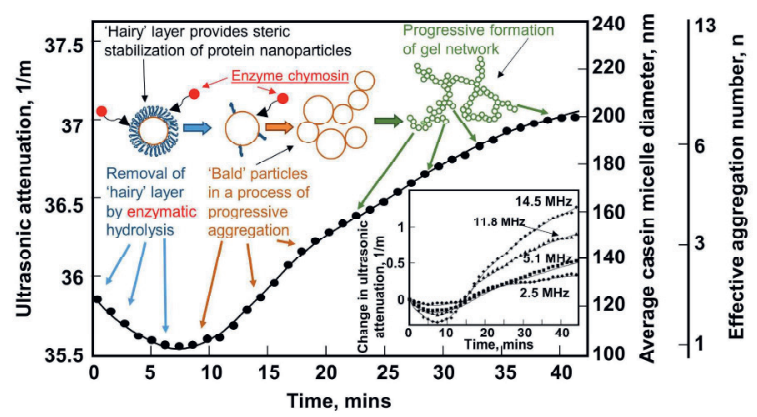

Fig. 4. Main frame: Ultrasonic attenuation measured at $14.5 \mathrm{MHz}$ in milk during the renneting process at $30{ }^{\circ} \mathrm{C}$. Insert: Effect of frequency on ultrasonic attenuation profiles measured during renneting of milk at $30{ }^{\circ} \mathrm{C}$ [8]. Adapted with permission from Dwyer C., Donnelly, L., Buckin, V., J. Dairy Res., 2005, 72(3), pp 303-310. Copyright (C Proprietors of Journal of Dairy Research 2005

Figure 4 represents real-time ultrasonic monitoring of enzymatic removal of 'hairy' layer of protein nanoparticles (casein micelles, 120 $\mathrm{nm}$ diameter), followed by particle aggregation and gelation milk at $30^{\circ} \mathrm{C}$ [8]. The k-casein 'hairy' layer provides steric stability of the particle dispersion, and its enzymatic removal is utilized in cheese making (renneting process). The measurements were performed in kinetic measuring regime of the HR-US 102 ultrasonic spectrometer, which simultaneously collected 
data measured at several pre-selected frequencies between 2 and $15 \mathrm{MHz}$. At time zero the enzyme (rennet, chymosin) was added to milk and $1 \mathrm{~mL}$ of the milk was placed in the ultrasonic cell. The ultrasonic attenuation profile was recalculated into the evolution of the average size of the protein particles during the process using the particle sizing module of HRUS 102 software and the physical parameters of the casein particles and the continuous medium [8]. It is interesting to note that particle aggregation (marked by increase in ultrasonic attenuation) occurs when the size of the particles is reduced by $20 \mathrm{~nm}$, which corresponds to the size of the 'hairy' layer [9].

\section{Dynamics of crystallization process.}

Figure 5 shows the real-time ultrasonic monitoring of crystallization of lysozyme initiated by addition of a precipitating agent to the ultrasonic cell containing $1 \mathrm{~mL}$ of $40 \mathrm{mg} / \mathrm{mL}$ solution of lysozyme in $0.1 \mathrm{M}$ sodium acetate buffer at $\mathrm{pH} 4.8$ at $20{ }^{\circ} \mathrm{C}$. Lysozyme is an antimicrobial enzyme produced by animals that forms part of the innate immune system. By varying the experimental conditions, various amounts and sizes of protein crystals can be produced.

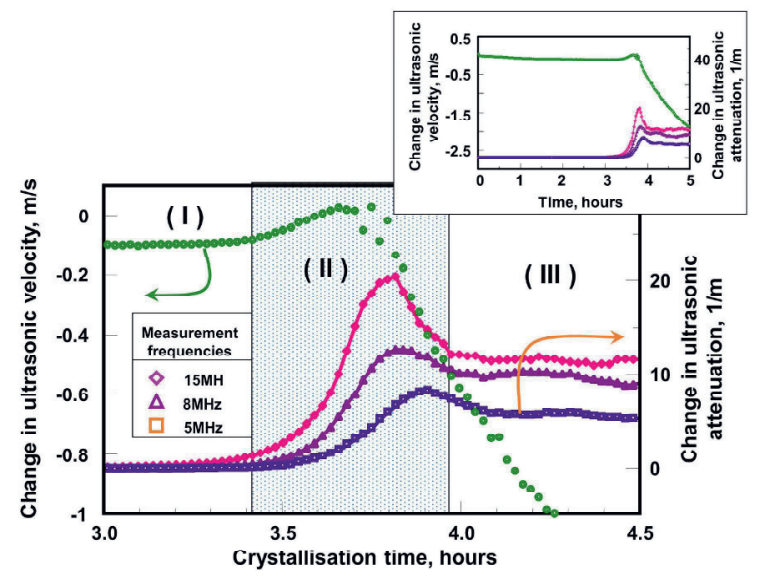

Fig. 5. Real-time monitoring of crystallization of lysozyme by high-resolution ultrasonic spectroscopy. Insert: the ultrasonic profile over the first 5 hours of crystallization process.

The obtained ultrasonic profiles show 3 stages in the crystallization process. Over the first 3.4 hours of the reaction, minor decrease in ultrasonic velocity is detected. At the end of Stage (I) the ultrasonic velocity and attenuation start to increase due to the formation of crystals. This increase continues through Stage (II) as the concentration and size of the crystals grow. The rise in ultrasonic velocity is caused by the increase in rigidity (decrease in compressibility) of the sample as a result of the formation of crystals. The rise of the ultrasonic attenuation can be attributed to scattering of the ultrasonic wave on the solid crystals formed. The scattering contribution is dependent on the ratio of crystal size and frequency (e.g. the attenuation at higher frequencies is more sensitive to the formation of small particles). During Stage (II) the ultrasonic velocity starts to decrease. In Stage (III) the ultrasonic attenuation nearly levels off indicating the end of crystal formation, however the velocity continues to decrease, as a consequence of the sedimentation of some percentage of crystals from the dispersion [10].

\section{Microemulsion phase diagrams.}

Figure 6 represents ultrasonic analysis of microemulsion phase diagrams utilized in the development of microemulsion based technologies. The presented phase diagram for IPM microemulsion (oil - isopropyl myristate, surfactant - Epikuron 200/cosurfactant - $n$ propanol (1:1), and water) made of pharmaceutical acceptable ingredients at $25{ }^{\circ} \mathrm{C}$ was obtained from titration profiles of ultrasonic velocity and attenuation. The titrations were performed by automatic stepwise additions of water to $1.5 \mathrm{~mL}$ of mixture of oil, surfactant and co-surfactant in the ultrasonic cell of HR-US 102SS ultrasonic spectrometer. An example of an ultrasonic titration profile in 20:80 w/w oil:surfactant/cosurfactant mixture is given in Figure 6. Overall, the ultrasonic titration profiles provide a number of parameters reflecting different levels of microstructural organization of the system $[11,12,13]$. The transitions between different states of the system are clearly identified by an abrupt change of concentration profile of ultrasonic velocity, attenuation and their frequency dependence. This includes the end of microemulsion phase, blue line, above which the mixture becomes opaque (phase IV). In addition to this, the ultrasonic titration profiles demonstrate a range of transitions below the blue line, which allowed upgrading the phase diagram measurable by other techniques with additional 'sub phases', Ia, Ib, II and III, within the part of the diagram below the blue line, called the microemulsion phase. The nature of these 'sub phases' can be related to the state of water and surfactant, including hydration water in the dispersed phase, hydration water in swollen reverse micelles and water in aqueous nano-size droplets surrounded by surfactant. Although, the details of interpretations of ultrasonic phase diagrams can be discussed, it is important to recognize that HR-US technique allows the identification of these different 'substates' on the phase diagram in simple titration measurements. Consideration of these states 


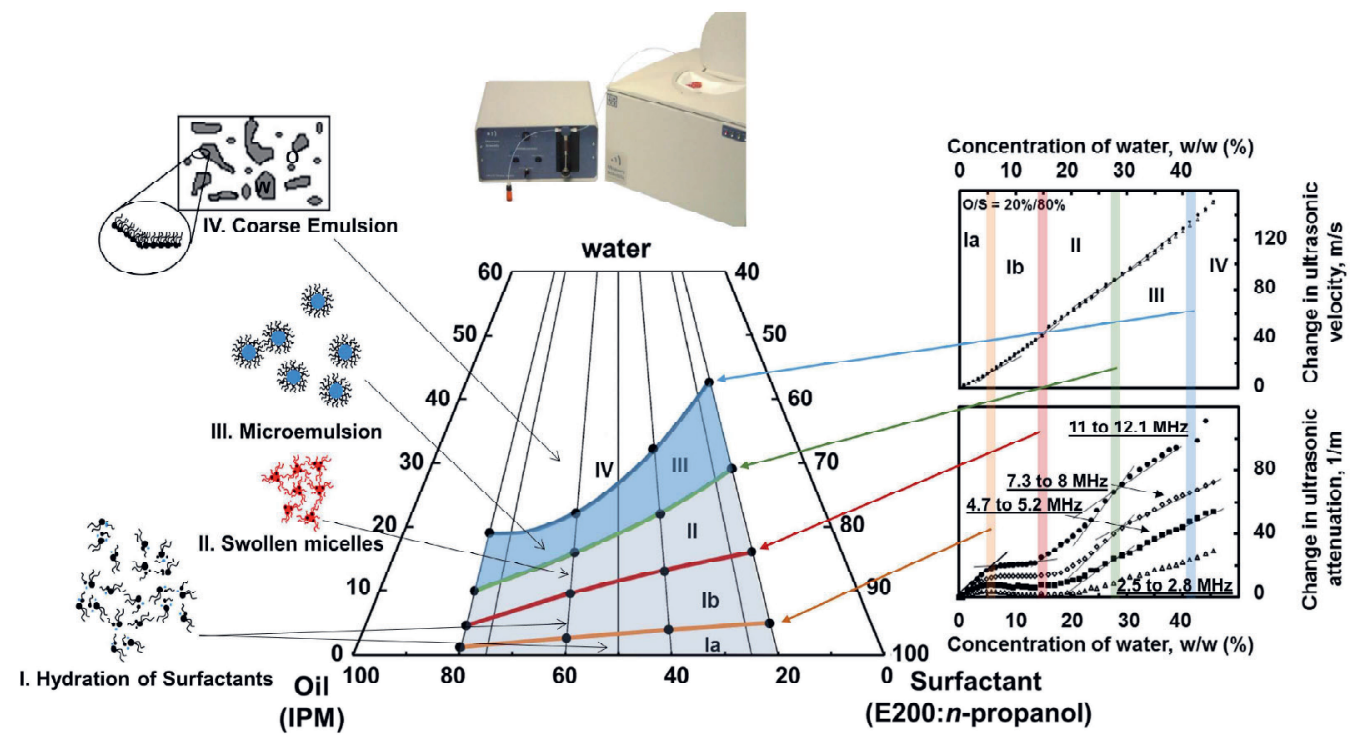

Fig. 6. Ultrasonic pseudo-ternary (concentration scale, $\% w / w)$ phase diagram of water/isopropyl myristate (IPM)/epikuron 200 (E200) and n-propanol (1:1) at $25{ }^{\circ} \mathrm{C}$. The transition lines between different sub-phases (hydration of surfactants, swollen micelles, microemulsion and coarse emulsion) represent the 'break' points on the ultrasonic (velocity and attenuation) titration profiles of surfactant/cosurfactant and oil mixture with water.

and their position on the phase diagram is important in design of w/o microemulsions designated for encapsulation of active ingredients (e.g. proteins) functioning of which requires an appropriate level of their hydration.

\section{Conclusions}

High-resolution ultrasonic spectroscopy is a powerful technique for real-time non-destructive analysis of molecular and microstructural transformations. As ultrasonic measurements characterize the properties of the bulk medium, the unwanted effects of surfaces, associated with reflectance spectroscopies and electrode techniques, are excluded. HR-US technique does not require optical activities, optical markers or other consumables. This, combined with the ability to perform measurements in opaque samples, broad temperature range, in static and flow-through regimes, in concentrated and diluted mixtures, allows application of this technique in a broad range of systems where other analytical methods often fail or have high analytical cost.

\section{References}

[1] M. J. W. Povey, T. J. Mason, Ultrasound in food processing, Springer Science \& Business Media (1998)

[2] V. Buckin, B. O'Driscoll, Ultrasonic waves and material analysis: recent advances and future trends, Lab Plus International 16(3), 17-21 (2002)

[3] E. P. P. Kis, Ultrasonic Velocity and Attenuation: Measurement Methods with Scientific and
Industrial Applications, Physical Acoustics V12: Principles and Methods 12, 277 (2012)

[4] V. Buckin, C. Smyth, High-resolution ultrasonic resonator measurements for analysis of liquids, Seminars in food analysis 4(2), 113-130, Aspen Publishers (1999)

[5] U. Kaatze, F. Eggers, K. Lautscham, Ultrasonic velocity measurements in liquids with high resolution-techniques, selected applications and perspectives, Measurement Science and Technology 19(6), 1-21 (2008); doi:10.1088/0957-0233/19/6/062001

[6] M. C. Altas, E. Kudryashov, V. Buckin, Ultrasonic Monitoring of Enzyme Catalysis; Enzyme Activity in Formulations for Lactose-Intolerant Infants. Analytical chemistry 88(9), 4714-4723 (2016); doi: 10.1021/acs.analchem.5b04673

[7] P. Resa, V. Buckin, Ultrasonic analysis of kinetic mechanism of hydrolysis of cellobiose by $\beta$ glucosidase, Analytical Biochemistry 415(1), 1-11 (2011); doi: 10.1016/j.ab.2011.03.003

[8] C. Dwyer, L. Donnelly, V. Buckin, Ultrasonic analysis of rennet-induced pre-gelation and gelation processes in milk, Journal of Dairy Research 72(3), 303-310 (2005); doi: $10.1017 /$ S0022029905001020

[9] C. Holt, D. G. Dalgleish, Electrophoretic and hydrodynamic properties of bovine casein micelles interpreted in terms of particles with an outer hairy layer, Journal of Colloid and Interface Science 114(2), 513-524 (1986); doi: 10.1016/0021-9797(86)90437-6.

[10] C. Smyth, B. O'Driscoll, J. Lawrence, S. Hickey, T. O'Reagan, V. Buckin, High-Resolution Ultrasonic Spectroscopy Analysis of 
Microemulsions, Pharmaceutical Technology Europe 16(6), 31-34 (2004)

[11] S. Hickey, M. J. Lawrence, S. A. Hagan, V. Buckin, Analysis of the phase diagram and microstructural transitions in phospholipid microemulsion systems using high-resolution ultrasonic spectroscopy, Langmuir 22(13), 55755583 (2006); doi: 10.1021/la052735t

[12] S. Hickey, S. A. Hagan, E. Kudryashov, V. Buckin, Analysis of phase diagram and microstructural transitions in an ethyl oleate/water/Tween 80/ Span 20 microemulsion system using high-resolution ultrasonic spectroscopy. International journal of pharmaceutics 388(1), 213-222 (2010); doi: 10.1016/j.ijpharm.2009.12.003

[13] V. Buckin, S. K. Hallone, Ultrasonic Characterisation of W/O Microemulsions Structure, Phase Diagrams, State of Water in Nano-Droplets, Encapsulated Proteins, Enzymes, 33-66, Intech (2012); doi:10.5772/36060 